\title{
ERRATA TO "HYPERGEOMETRIC OPERATOR SERIES AND RELATED PARTIAL DIFFERENTIAL EQUATIONS”
}

\author{
LOUIS R. BRAGG
}

I would like to make the following corrections to my paper Hypergeometric operator series and related partial differential equations, Trans. Amer. Math. Soc. 143 (1969), 319-336.

On p. 333. Example 4 should carry the title $A$ Dirichlet problem.

On $p$. 333. In the line above (5.8), the name Beltrami should be replaced by GASPT.

On p. 334. In the 6th line down, the statement "This result was obtained earlier by J. B. Diaz [13]," should be replaced by

"Except for a constant multiplier, this result agrees with one obtained earlier by A. Weinstein in [13]. (In formula (4.1), p. 109 of that paper, replace $x_{i}$ by $x_{i}-\xi_{i}$, $i=1, \ldots, m$, and choose $b=0$.)"

On p. 336. The new reference [13] should read as follows:

13. A. Weinstein, The singular solutions and the Cauchy problem for generalized Tricomi equations, Comm. Pure Appl. Math. 7 (1954), 105-116. MR 16, 137.

OAKLAND UNIVERSITY,

ROCHESTER, MichigaN 48063 